\title{
A Reliable and Sustainable Multicomponent Access to Protein Degraders
}

Irene Preet Bhela ${ }^{1}$, Alice Ranza ${ }^{1}$, Marta Serafini ${ }^{1,2}$ and Tracey Pirali ${ }^{1 *}$

${ }^{1}$ Department of Pharmaceutical Sciences, Università degli Studi del Piemonte Orientale, Largo Donegani 2, 28100 Novara, Italy

${ }^{2}$ Department of Chemistry, Chemistry Research Laboratory, University of Oxford, Mansfield Road, Oxford OX1 3TA, UK

E-mail: tracey.pirali@uniupo.it

\section{Graphical abstract}

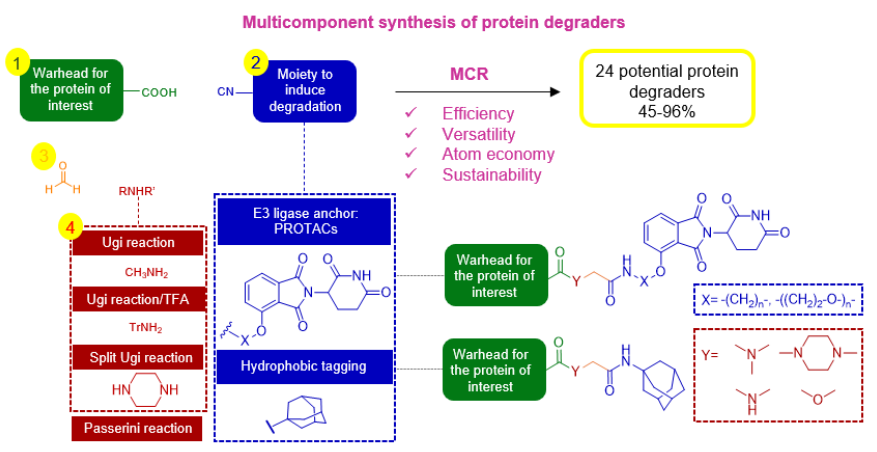

\section{Abstract}

The use of small molecules to induce targeted protein degradation is increasingly growing in the drug discovery landscape and protein degraders have progressed rapidly through the pipelines. Despite the advances made so far, their synthesis still represents a significant burden and new approaches are urgently demanded. Herein, we report an unprecedented platform that leverages the modular nature of both multicomponent reactions and degraders to enable the preparation of highly decorated PROTACs and hydrophobic tag-mediated degraders. Compared to the existing methods, our 
approach offers a versatile and cost-effective means to access libraries of protein degraders and increase the chance of identifying successful clinical candidates.

\section{Keywords}

PROTACs; protein degraders; multicomponent reactions; Ugi reaction. 
Protein degraders represent a new strategy to reduce expression of disease-related proteins and are set to revolutionize drug discovery ${ }^{1}$. Among them, PROTACs (PRoteolysis TArgeting Chimeras) have drawn attention as one of the most appealing approaches ${ }^{2}$. Being composed of a ligand for the protein of interest (POI) (referred to as warhead), a linker and a ligand for the E3 ubiquitin ligase (referred to as anchor), PROTACs are able to hijack the ubiquitin-proteasome cascade and force the target protein to be degraded (Figure 1). Upon completion of the cycle, they are still available for successive rounds of degradation, thus minimizing the need for a continuous drug exposure ${ }^{3}$. Over one hundred different proteins have been targeted so far, and even those proteins traditionally considered "undruggable" have been shown to be degraded by this system ${ }^{4}$. Noteworthy, compared to occupancy-driven approaches, PROTACs allow to abrogate all the activities associated with the selected protein. Their potential to knock down disease-related proteins is exemplified by the first degraders that have reached human clinical trials ${ }^{5-7}$.

From a structural point of view, the warhead is represented by a ligand that binds the POI, independently from its mechanism of action. Concerning the anchor for the E3 ligases, cereblon (CRBN) targeting ligands (i.e. IMiD-based ligands, such as thalidomide and its structural analogues $)^{8}$ and Von Hippel-Lindau (VHL) targeting ligands (i.e. hydroxyproline-based molecules) ${ }^{3}$ are the most commonly used binders. Given that there is relatively limited room of manoeuvre to alter the warhead and the anchor, synthetic modifications are focused on both the linker, that can be of different length and composition (i.e. linear aliphatic linkers, PEG and extended glycol chains), and the linkage points, whose chemical composition is usually driven by the chemical reactions used to assemble the PROTAC rather than a rational choice (i.e. amines, ethers, amide moieties). The sites of attachment of the linker to the anchor and the warhead are critical and are usually selected by analyzing the solvent-exposed regions, in order to minimize the interference of the linker with the binding of the PROTAC to the E3 ligase and the POI, respectively (Figure 1). Only when all these structural requirements are found in the optimal combination, they engage in the productive formation of the ternary E3 ligase-PROTAC-POI complex and activate the proteasome cascade ${ }^{9,10}$. 
Fig. 1 - Representative structure of a PROTAC, its conventional synthesis and the MCR platform.

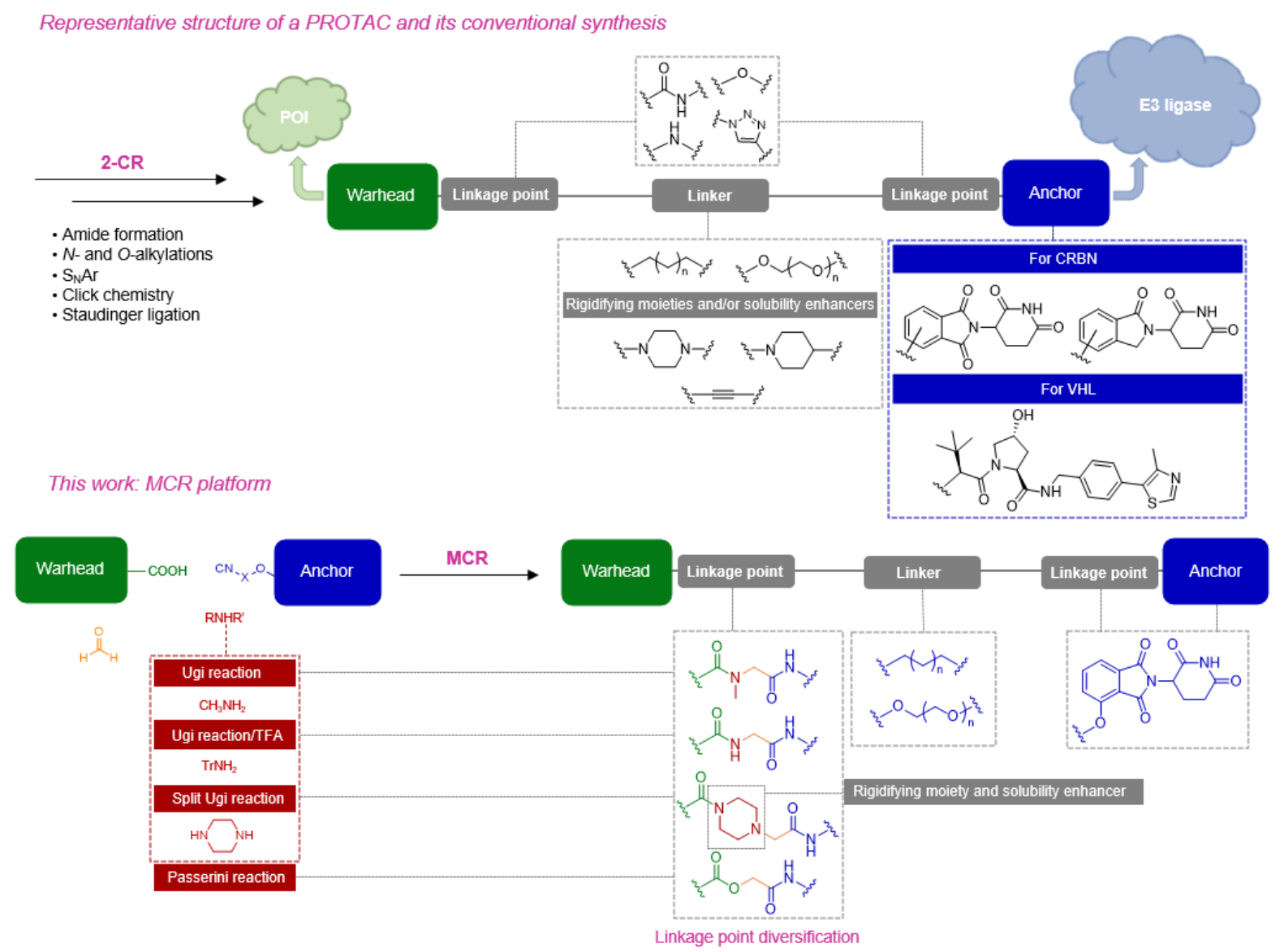

The structural complexity of the ternary complex (TC) makes it a formidable challenge to predict $a$ priori which combination of the different synthons is the best one to achieve degradation of the POI, even if X-ray crystal structures, molecular modelling and dynamics might at times be of help ${ }^{11-13}$. Besides degradation efficiency, similarly to any other medicinal chemistry campaign, properties such as solubility, metabolic stability and cell permeability represent an additional challenge to achieve an optimal in vivo effect, with far more reason since PROTACs lie outside the rule-of-five space ${ }^{14-16}$. Hence, efforts have been devoted to rationally explore the role of the different structural elements in conferring favorable pharmacokinetic properties and a special attention has been given to the chemistry of the linker, whose optimization has a crucial impact on the ADME profile throughout the R\&D process ${ }^{14,17,18}$. Linear aliphatic and PEG-based linkers are commonly used in the early phases of development to assess the most favorable length to form an efficient ternary complex, while at a 
later stage they are usually refined and enriched with rigidifying motifs (e.g. piperazines, piperidines) to further improve pharmacokinetic parameters (Figure 1) ${ }^{19}$.

Despite the advances made so far in the rational design of PROTACs, the conversion of a ligand for the POI into an effective degrader in vivo remains rather empirical and based on a "trial and error" approach $^{20}$. Extensive investigation of multiple warheads, anchors, linker types and lengths, and linkage points is needed from the early stage of PROTAC development. Nevertheless, the lack of reliable and economically sustainable synthetic methods represents an important caveat that necessarily limits the number of accessible compounds. The assemble of PROTACs has proven to be far from straightforward as it involves the asymmetric diversification of the two sides of the linker and their chemoselective reactions with the two protein binding motifs. This challenge is usually achieved through orthogonal conditions or protection/deprotection sequences. Linking strategies to couple the linker with the warhead and the anchor include amide bond formation, $\mathrm{N}$ - and $\mathrm{O}$ alkylations, nucleophilic aromatic substitution, acylation and Williamson ether synthesis, transformations that are known to suffer from poor reactivity, chemoselectivity and atom economy (Figure 1$)^{19}$. It results that the preparation of PROTACs requires low-yielding and cumbersome multistep synthetic routes, makes extensive use of protecting groups, and relies on highly functionalized and costly building blocks.

To overcome these limitations, notable advances aimed at simplifying the access to protein degraders have been reported ${ }^{18}$ and include the use of orthogonally protected bifunctional linkers ${ }^{21}$, solid phase synthesis $^{22}$, click chemistry ${ }^{23}$, Staudinger ligation ${ }^{24}$ and others ${ }^{25}$. Nevertheless, none of these strategies has established itself as the ideal method so far, due to existing limitations. For instance, among the recent methods reported to ease the preparation of PROTACs, the click chemistry platform allows the preparation of both CRBN and VHL recruiting degraders, but the introduction of the triazole ring is well-known for its reluctance to scale-up ${ }^{26}$. Despite the advances made so far, the synthesis of PROTACs still represents a significant synthetic burden, limiting the chemical space that can be accessed. 
In an effort to streamline the discovery of successful PROTACs, we report a modular synthetic platform that capitalizes on the versatility of multicomponent reactions (MCRs) to assemble heterobifunctional protein degraders (Figure 1).

\section{Results}

We initially focused on the Ugi reaction ${ }^{27,28}$, that stands out for its efficiency, versatility, high atom economy, and simple and environmentally friendly experimental procedures, where the only byproduct is one water molecule per molecule of product.

\section{Selection of synthons for the Ugi reaction.}

For an initial proof of concept, we decided to focus on the following synthons as a model system: five different isocyanides bearing the thalidomide-based CRBN recruiting anchor (12-16, Figure 2), a carboxylic acid (17) bearing the warhead based on (+)-JQ1 (a well-known BET bromodomain inhibitor), formaldehyde (18) as the carbonyl compound to minimize the interference of the linker with the binding to the POI and three different commercially available amines (methylamine 19, tritylamine as surrogate of ammonia 25, and piperazine 31) (Figure 3). All these building blocks are commercially available, except for the thalidomide-based recruiting moiety, that can be easily prepared as previously described ${ }^{23}$, and the isocyanides, synthesized following an in-house developed synthetic route.

\section{Preparation of the linkers.}

In the first step, an amino alcohol (1-5, Figure 2) is treated with ethyl formate and, upon completion of the reaction and after evaporation of the formylating agent, the resulting formamide is treated with tosyl chloride in the presence of triethylamine to give the heterobifunctional linker (6-10) bearing both the isocyanide and the tosylate in 57-73\% yield. According to a recent report by Meier et al. published on Green Chemistry, tosyl chloride represents an efficient and practical alternative to toxic and hazardous dehydrating agents such as phosphorous oxychloride in the preparation of aliphatic isocyanides ${ }^{29}$. This method is perfectly suited to our purpose as it can simultaneously dehydrate the 
formamide to isocyanide and tosylate the hydroxyl group ${ }^{29}$. The third reaction is an $O$-alkylation that takes place by adding derivatives 6-10 to the thalidomide bearing a hydroxyl group at 4-position (11) in the presence of sodium bicarbonate. Notably, undesired substitution to the glutarimide ring imide nitrogen, that would result in the loss of E3 ligase recruitment, does not occur under our optimized conditions.

By this strategy, both linear aliphatic (12-14) and PEG (15-16) linkers of different length have been prepared in yields spanning from $42 \%$ to $81 \%$. The proposed sequence stands out for its sustainability: all the required reagents (from ethyl formate to sodium bicarbonate) are cheap and offer a simplified protocol to access the required linkers. Furthermore, as the isocyanides are stable upon long-term storage $^{30}$, they can be prepared and stocked to produce "user friendly" tool kits for the synthesis and diversification of PROTACs. Noteworthy, they do not suffer from the unpleasant smell typical of the isocyanides, probably due to the high molecular weight and solid state at room temperature.

With these linkers in our hands, we turned our efforts into the Ugi reaction, and we started investigating its use in PROTACs synthesis by varying the amine component.

Fig. 2 - Synthesis of the isocyanide-based library of CRBN recruiting anchors.

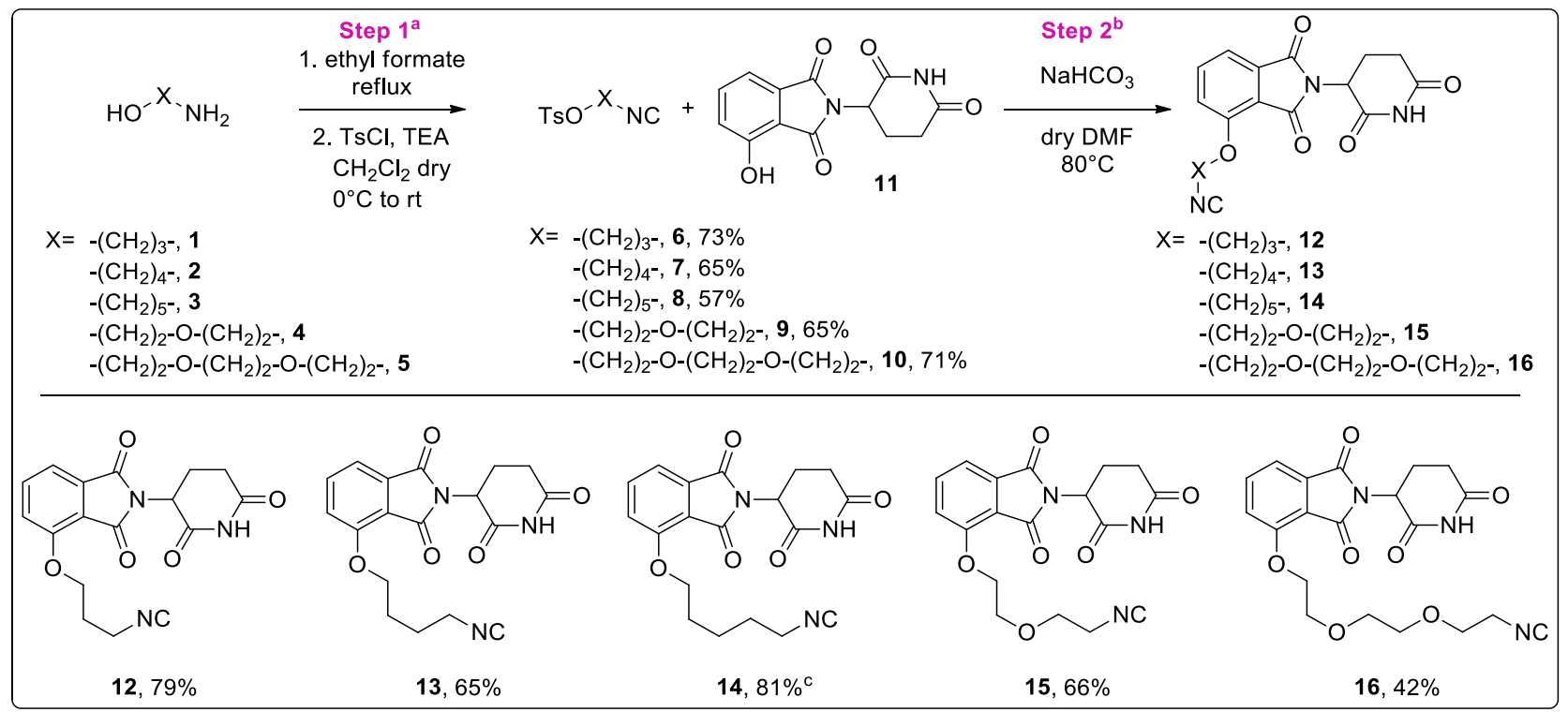

\footnotetext{
${ }^{\text {a }}$ Reactions were conducted on a $20.0 \mathrm{mmol}$ scale with respect of 1-5. Yields over two steps are given. Conditions: 1.
} Reactions were carried out with ethyl formate $(13 \mathrm{~mL})$ at reflux for $6 \mathrm{~h}$. 2. Reactions were carried out with $\mathrm{TsCl}(22.5$ 
mmol, 3 equiv), TEA (45.0 mmol, 6 equiv), and dry $\mathrm{CH}_{2} \mathrm{Cl}_{2}(14 \mathrm{~mL})$ at room temperature for 5 h. ${ }^{\mathrm{b}}$ Reactions were conducted on a $2.2 \mathrm{mmol}$ scale with respect of 11. Conditions: Reactions were carried out with $\mathrm{NaHCO}_{3}(4.4 \mathrm{mmol}, 2$ equiv) and dry DMF $(6 \mathrm{~mL})$ at $80{ }^{\circ} \mathrm{C}$ overnight. ${ }^{\mathrm{c}}$ This reaction was scaled up on a $6.0 \mathrm{mmol}$ scale with respect of 11.

\section{Different amines to probe the warhead linkage point.}

We began by methylamine (19) as a model for conventional primary amines and PROTACs 20-24 (Figure 3A) were synthesized in 54-75\% yields. The products were purified by conventional flash chromatography, without need for preparative HPLC techniques.

In order to further reduce steric hindrance at the attachment point and minimally influence affinity of the warhead for its target protein, we then turned to explore the use of tritylamine (25), that our laboratory had recently reported as an effective surrogate of ammonia ${ }^{31}$, especially when coupled with formaldehyde as the carbonyl component. The reaction was performed in methanol and, upon completion, the trityl group was cleaved by adding trifluoroacetic acid to give the desired PROTACs 26-30 in 55-82\% yields (Figure $3 \mathrm{~B}$ ).

Finally, we exploited the split Ugi reaction ${ }^{32}$ that is performed using a secondary amine, instead of a primary amine: one $\mathrm{N}$-atom is acylated, while the other one is alkylated in one step, without the need for coupling agents or protecting groups. This variant particularly suites our purposes as it allows the one-pot introduction of the piperazine ring, a privileged substructure in PROTACs as a solubility enhancer, as a means to improve metabolic stability ${ }^{14}$ and as a rigid moiety that leads to a stable ternary complex formation ${ }^{33-35}$. Noteworthy, the use of piperazine as the amine component allows for the increase of $\mathrm{sp}^{3}$ character and the reduction of the number of HBDs and HBAs that have been associated with improved oral drug delivery. This is exemplified by the recently disclosed structures of ARV-110 for metastatic castration-resistant prostate cancer and ARV-471 for metastatic breast cancer that are two orally available PROTACs currently in clinical trials (NCT0888612 and NCT04072952) ${ }^{36}$. By means of the split Ugi reaction, PROTACs 32-36 were synthesized, with yields of $63-82 \%$ (Figure $3 \mathrm{C}$ ). 
Fig. 3 - Library of PROTACs synthesized by varying the amine group in the Ugi MCR.

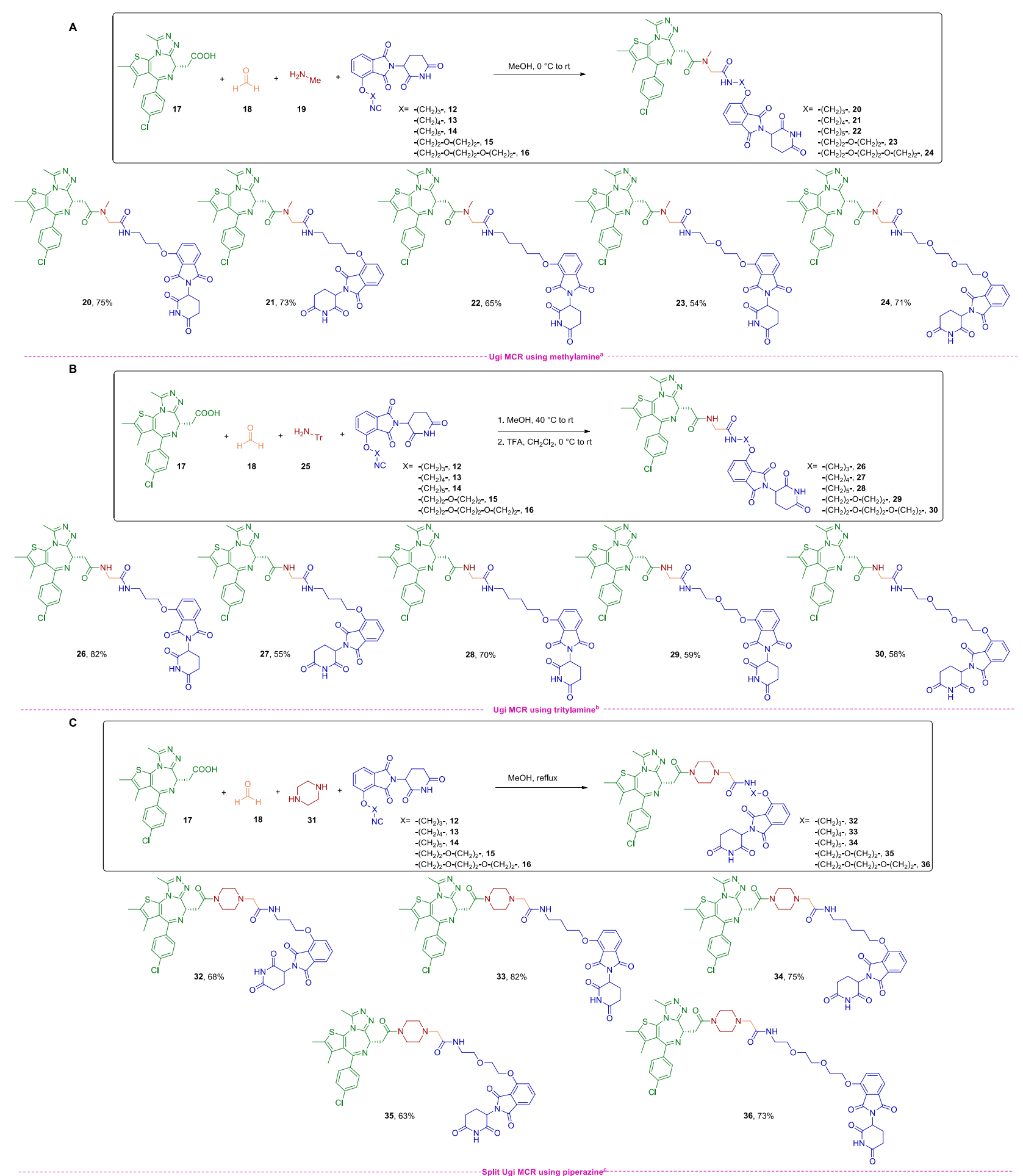

${ }^{\text {A }}$ Reactions were conducted on a $0.18 \mathrm{mmol}$ scale with respect of 12-16. Conditions: Reactions were carried out with $(+)-$ JQ1 (0.12 mmol, 1 equiv), formaldehyde (37\% aqueous solution stabilized with 7\% methanol, $0.36 \mathrm{mmol}, 3$ equiv), methylamine ( $40 \%$ aqueous solution, $0.24 \mathrm{mmol}, 2$ equiv), and $\mathrm{MeOH}(0.50 \mathrm{~mL})$ at $0{ }^{\circ} \mathrm{C}$ for $2 \mathrm{~h}$, then at room temperature for $1 \mathrm{~h} .{ }^{\text {B }}$ Reactions were conducted on a $0.12 \mathrm{mmol}$ scale with respect of 12-16. Yields over two steps are given. Conditions: 1. Reactions were carried out with (+)-JQ1 ( $0.12 \mathrm{mmol}, 1$ equiv), paraformaldehyde $(0.24 \mathrm{mmol}, 2$ equiv), 
tritylamine (0.12 mmol, 1 equiv), and $\mathrm{MeOH}(0.5 \mathrm{~mL})$ at $40{ }^{\circ} \mathrm{C}$ for $40 \mathrm{~min}$, then overnight at room temperature. 2. Reactions were carried out with TFA $(256 \mu \mathrm{L})$, and $\mathrm{CH}_{2} \mathrm{Cl}_{2}(256 \mu \mathrm{L})$ at $0{ }^{\circ} \mathrm{C}$ for $30 \mathrm{~min}$, then at room temperature for 3 h. ${ }^{\mathrm{C}}$ Reactions were conducted on a $0.12 \mathrm{mmol}$ scale with respect of 12-16. Reactions were carried out with $(+)-\mathrm{JQ} 1(0.12$ mmol, 1 equiv), paraformaldehyde $(0.12 \mathrm{mmol}, 1$ equiv $)$, piperazine $(0.12 \mathrm{mmol}, 1$ equiv), and $\mathrm{MeOH}(0.50 \mathrm{~mL})$ heating at reflux for $2 \mathrm{~h}$. Color code does not refer to the MCR mechanism, but is intended to represent the synthons and their assemble into the protein degraders.

\section{Different carboxylic acids to probe the warhead.}

With the aim of covering a large chemical diversity, we turned our efforts to exploring whether carboxylic acids other than the (+)-JQ1 based could be accommodated in the platform and carboxylic acids based on olaparib (poly(ADP-ribose) polymerase PARP inhibitor) and CX4945 (casein kinase CK2 inhibitor) scaffolds were selected. In spite of the fact that reactions appeared slower and less clean compared to (+)-JQ1, due to poor solubility of the two carboxylic acids in methanol, three additional PROTACs (39-41) were synthesized, in yields comprised between 45 and 60\% (Figure 4A).

\section{Passerini reaction.}

Very recently, Ciulli et al. have reported that the bioisosteric replacement of the amide with an ester at the linkage point between the warhead and the linker might enhance the pharmacokinetic and pharmacodynamic performance of the PROTAC, without affecting its plasma stability ${ }^{37}$. We therefore investigated the inclusion of the Passerini reaction ${ }^{38}$ in the MCR platform and demonstrated that the isocyanide-bearing linkers 12-14 react well in the absence of the amine component and in dichloromethane as reaction solvent to afford $\mathbf{4 2 - 4 4}$ in $53-71 \%$ yield (Figure $4 \mathrm{~B}$ ).

\section{Hydrophobic tagging.}

Finally, we leveraged the multicomponent approach to prepare hydrophobic tagging (HyT), a targeted proteolytic system similar to PROTACs ${ }^{1}$. These heterobifunctional degraders display a highly lipophilic moiety, such as an adamantane, linked to a ligand for the $\mathrm{POI}^{39,40}$. Upon binding to the protein target, the HyT mimics a partially denatured protein folding state, activating the unfolded 
protein response and inducing degradation of the POI, even if the precise mechanism has not been fully elucidated yet ${ }^{41}$. To this aim, we used commercially available adamantyl isocyanide (45) and prepared in a one-pot fashion three HyTs by the Ugi reaction and its variants (46-48, Figure 4C), in $70-96 \%$ yield.

Fig. 4 - Changing the carboxylic acid, the MCR and the protein degrader subtype.

A
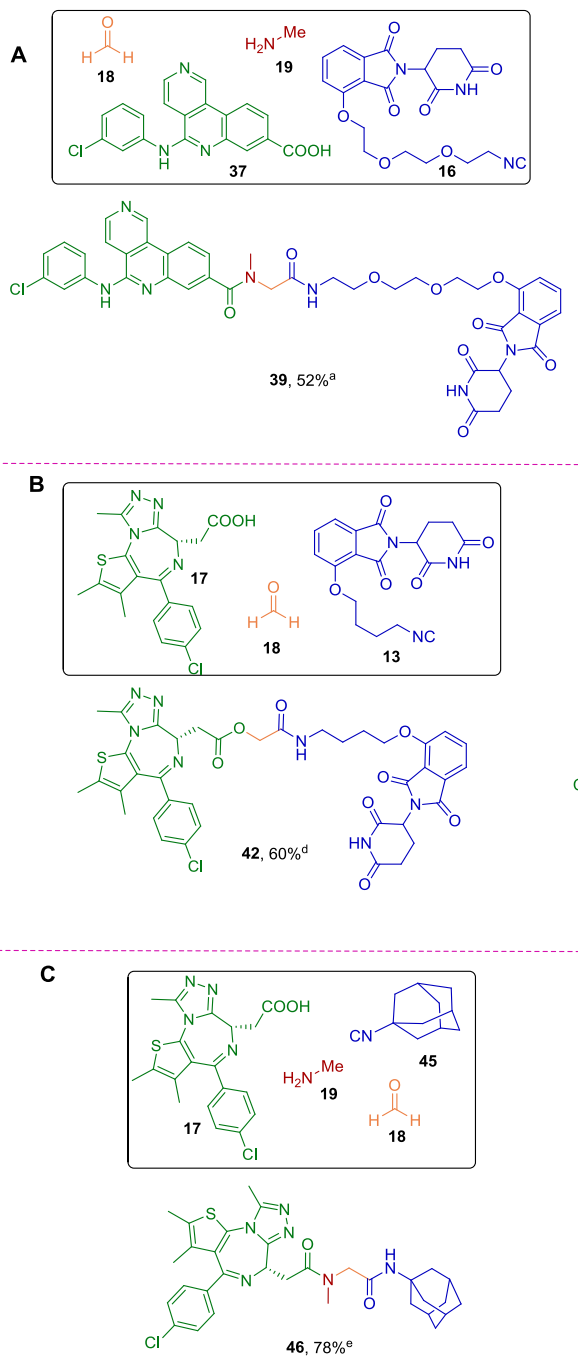
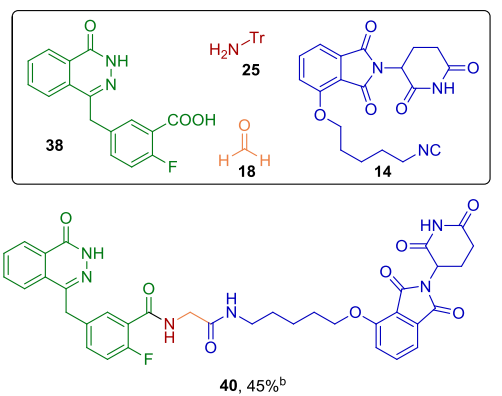

$40,45 \%$
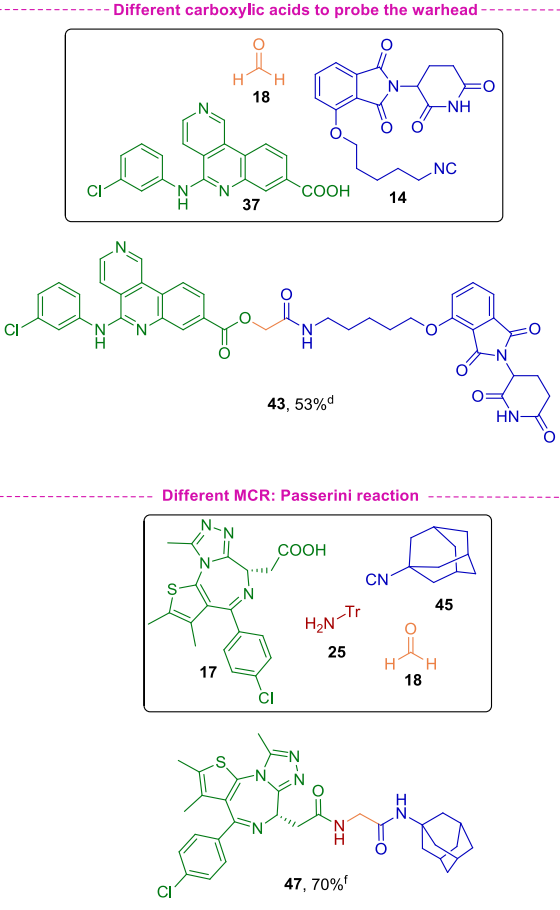

Hydrophobic taggin
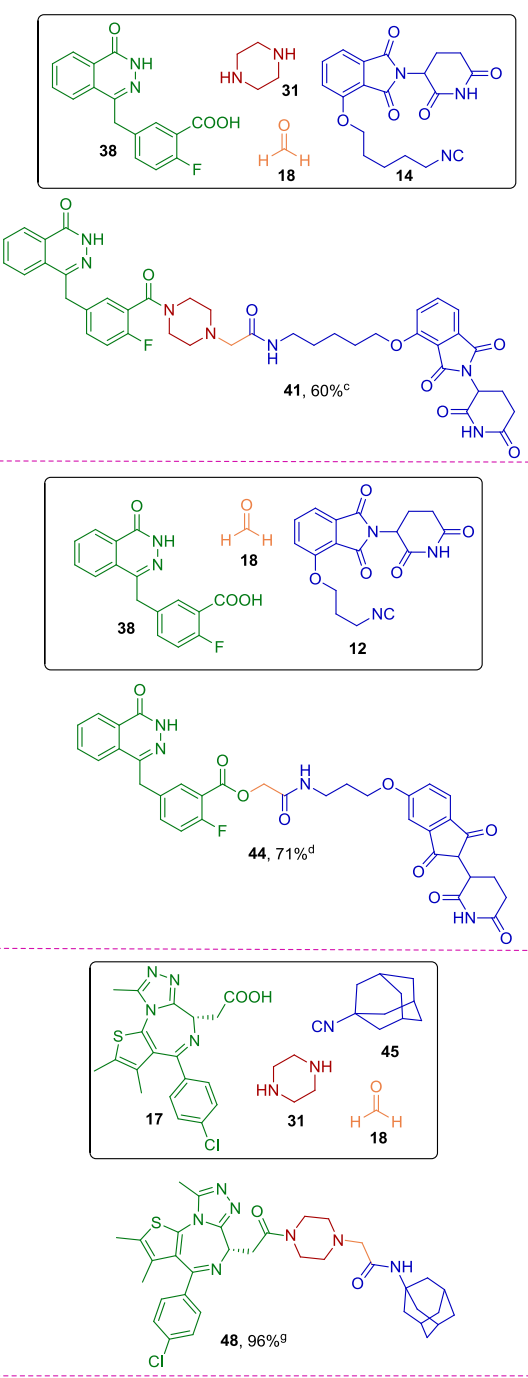

a Reaction was conducted on a $0.18 \mathrm{mmol}$ scale with respect of 16. Conditions: Reaction was carried out with formaldehyde (37\% aqueous solution stabilized with $7 \%$ methanol, $0.36 \mathrm{mmol}, 3$ equiv), methylamine (40\% aqueous solution, $0.24 \mathrm{mmol}, 2$ equiv), carboxylic acid $\left(0.12 \mathrm{mmol}, 1\right.$ equiv), and $\mathrm{MeOH}(0.50 \mathrm{~mL})$ at $0{ }^{\circ} \mathrm{C}$ for $2 \mathrm{~h}$, then at room temperature overnight. ${ }^{\mathrm{b}}$ Reaction was conducted on a $0.12 \mathrm{mmol}$ scale with respect of $\mathbf{1 4}$. Yields over two steps are given. Conditions: 1 . Reaction was carried out with paraformaldehyde ( 0.24 mmol, 2 equiv), tritylamine $(0.12 \mathrm{mmol}, 1$ equiv), carboxylic acid $\left(0.12 \mathrm{mmol}, 1\right.$ equiv), and $\mathrm{MeOH}(0.50 \mathrm{~mL})$ at $40{ }^{\circ} \mathrm{C}$ for $40 \mathrm{~min}$, then at $50{ }^{\circ} \mathrm{C}$ for $2 \mathrm{~h} .2$. Reaction 
was carried out with TFA $(384 \mu \mathrm{L})$, and $\mathrm{CH}_{2} \mathrm{Cl}_{2}(384 \mu \mathrm{L})$ at $0{ }^{\circ} \mathrm{C}$ for $30 \mathrm{~min}$, then at room temperature for $3 \mathrm{~h} .{ }^{\mathrm{c}} \mathrm{Reaction}$ was conducted on a $0.12 \mathrm{mmol}$ scale with respect of 14 . Reaction was carried out with paraformaldehyde $(0.12 \mathrm{mmol}, 1$ equiv), piperazine ( $0.12 \mathrm{mmol}, 1$ equiv), carboxylic acid ( $0.12 \mathrm{mmol}, 1$ equiv), and $\mathrm{MeOH}(0.50 \mathrm{~mL})$ heating at reflux for $2 \mathrm{~h} .{ }^{\mathrm{d}}$ Reactions were conducted on a $0.24 \mathrm{mmol}$ scale with respect of $\mathbf{1 3}$. Reactions were carried out with formaldehyde ( $37 \%$ aqueous solution stabilized with $7 \%$ methanol, 0.48 mmol, 4 equiv), carboxylic acid (0.12 mmol, 1 equiv), and $\mathrm{CH}_{2} \mathrm{Cl}_{2}(1.30 \mathrm{~mL})$ heating at $40{ }^{\circ} \mathrm{C}$ for $4 \mathrm{~h} .{ }^{\mathrm{e}}$ Reactions were conducted on a $0.12 \mathrm{mmol}$ scale with respect of 45. Conditions: Reactions were carried out with formaldehyde (37\% aqueous solution stabilized with $7 \%$ methanol, 0.24 mmol, 2 equiv), methylamine (40\% aqueous solution, 0.14 mmol, 1.2 equiv), (+)-JQ1 (0.12 mmol, 1 equiv), and MeOH $(0.50 \mathrm{~mL})$ at $0{ }^{\circ} \mathrm{C}$ for $2 \mathrm{~h}$, then at room temperature for $1 \mathrm{~h} .{ }^{\mathrm{f}}$ Reaction was conducted on a 0.12 mmol scale with respect of 45. Yields over two steps are given. Conditions: 1 . Reaction was carried out with paraformaldehyde $(0.24 \mathrm{mmol}, 2$ equiv), tritylamine (0.12 mmol, 1 equiv), (+)-JQ1 (0.12 mmol, 1 equiv), and $\mathrm{MeOH}(0.50 \mathrm{~mL})$ at $40{ }^{\circ} \mathrm{C}$ for $40 \mathrm{~min}$, then overnight at room temperature. 2. Reaction was carried out with TFA $(384 \mu \mathrm{L})$, and $\mathrm{CH}_{2} \mathrm{Cl}_{2}(384 \mu \mathrm{L})$ at $0{ }^{\circ} \mathrm{C}$ for $30 \mathrm{~min}$, then at room temperature for $3 \mathrm{~h} .{ }^{\mathrm{g}}$ Reaction was conducted on a $0.12 \mathrm{mmol}$ scale with respect of $\mathbf{4 5}$. Reaction was carried out with paraformaldehyde $(0.12 \mathrm{mmol}, 1$ equiv), piperazine $(0.12 \mathrm{mmol}, 1$ equiv), (+)-JQ1 (0.12 mmol, 1 equiv), and $\mathrm{MeOH}(0.50 \mathrm{~mL})$ heating at reflux for $2 \mathrm{~h}$. Color code does not refer to the MCR mechanism, but is intended to represent the synthons and their assemble into the protein degraders.

\section{Discussion}

In this study, we present an unprecedented MCR-based platform that enables the expedient synthetic entry into the chemical space of protein degraders in the scope of green chemistry. When compared to the existing two-components methods, our approach stands out for being:

Reliable, high yielding, protecting group-free and stereoconservative. To give an idea of the simplicity of the approach, in a relatively short period of time, we synthesized a library of 24 highly decorated potential protein degraders with an average yield of $70 \%$. The library was prepared starting from a set of CRBN recruiting isocyanides that were easily synthesized in two steps (i.e. formylation of an aminoalcohol followed by simultaneous tosylation and dehydration).

Our platform obviates the need to perform protection/deprotection steps typically required in the synthesis of PROTACs, except for the tritylamine when used as a surrogate of ammonia. An additional feature is given by the well-known stereoconservative nature of Ugi and Passerini reactions 
that is fundamental when chiral warheads and anchors are involved in the assemble of the protein degrader ${ }^{42,43}$.

Versatile. By varying the amine component in the MCR, the linkage point between the warhead and the linker is highly diversified, allowing for extensive structure-activity relationship and structureproperty relationship studies around not only the linker, its length and composition, but also the attachment point to the warhead, that strongly influences the affinity of the ligand for the POI and is responsible for metabolic instability ${ }^{14}$. We have applied this approach to the synthesis of both PROTACs and hydrophobic tagging, depending on the nature of the isocyanide used to perform the MCR, using as model systems different warheads for three POI. Nevertheless, the versatility of MCRs might find other applications, for example in the design of dual or trivalent PROTACs. In the first strategy, two different ligands for two POIs compose the chimeric molecule to allow for the simultaneous degradation of two proteins in one fashion ${ }^{44}$. In the second, two ligands for the same POI are fused together to engage a more cooperative ternary complex and boost the degradation of the $\mathrm{POI}^{45}$. As in both strategies the two distinct ligands are tethered to the E3 ligase-recruiting moiety via a branched linker, either the carbonyl or the primary amine components of the MCR could potentially be further functionalized to act as warhead for the second POI.

Sustainable and cost-effective. The process is characterized by a low environmental footprint. Moreover, except for the (+)-JQ1 based carboxylic acid, all the other substrates required for the preparation of both the linkers and the final degraders are inexpensive and easy handling, in stark contrast with the cost associated with most of the synthons that are currently commercially available ${ }^{46}$.

The above advantages become evident when comparing the overall synthetic process required for the synthesis of the PROTAC dBET21 $1^{47,48}$ and its counterpart 22, that shares the same linker length while displaying one additional amide moiety (Table 1). The patented route to dBET21 requires five steps with an overall yield of 3\%, in stark contrast with the MCR platform that allows the synthesis of 22 in four steps with a ten-fold higher yield (30\%). The difference mainly relies on the last step that links 
the warhead to the linker: while the Ugi reaction affords the product in a $65 \%$ yield after with an atom economy of $73 \%$, the last step for the synthesis of dBET 21 is an amide formation reaction mediated by HATU that suffers from a very low yield (10\%) and poor atom economy (46\%). A similar situation is found when comparing the olaparib-based PROTAC ${ }^{14}$ and $\mathbf{4}$, where the piperazine ring is inserted during the split Ugi reaction (Table 1). While in the examples reported in the literature the last reaction requires at least one purification step by preparative HPLC or TLC to give the final compound, in the preparation of our products no further technique was necessary besides column chromatography.

Table 1 - Comparative table between previously reported synthetic approaches to PROTACs and this work.

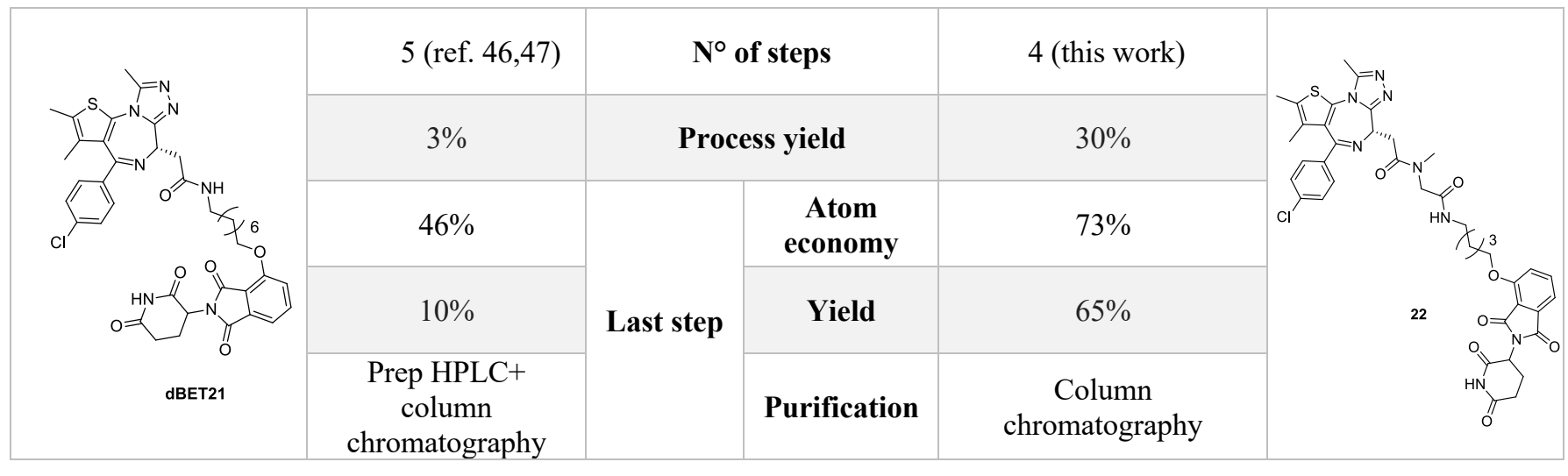

\begin{tabular}{|c|c|c|c|}
\hline & 3 (ref. 14) & No of steps & 4 (this work) \\
\hline
\end{tabular}

For the time being, we have only scraped the surface of the potential of the platform in the protein degraders field: the described variants have manifold applications and can be easily applied to other proteins for which ligands are available. Indeed, the reported method is suitable for different warheads, where the only pre-requisite is the presence of a carboxylic moiety. Making protein 
degraders easily accessible to chemists at a reasonable cost must be a major objective in the time to come and we envision that our method will help to achieve the goal.

\section{Methods}

Materials. Commercially available reagents and solvents were used as purchased from Zentek, Sigma-Aldrich, MedChemExpress and Carlo Erba without further purification. When needed, solvents were distilled and stored on molecular sieves. Reactions were monitored by thin layer chromatography (TLC) carried out on $5 \mathrm{~cm} \times 20 \mathrm{~cm}$ silica gel plates with a layer thickness of 0.25 $\mathrm{mm}$, using UV light as a visualizing agent. When necessary, TLC plates were visualized with aqueous $\mathrm{KMnO}_{4}$ and Dragendorff reagent.

Instrumentations. NMR spectra were recorded on a Bruker Avance $\mathrm{Neo} 400 \mathrm{MHz}\left({ }^{1} \mathrm{H}\right.$ and ${ }^{13} \mathrm{C} 400$ and $101 \mathrm{MHz}$, respectively) in appropriate deuterated solvents. All protein degraders based on (+)JQ1 structure were obtained as a mixture of diastereomers which include racemic mixture at the IMiD stereocenter with enantiopure (+)-configuration at the JQ1 stereocenter. IR and HRMS spectra were obtained using FT-IR Bruker Alpha II and (Thermo Fisher Q-Exactive Plus) equipped with an Orbitrap (ion trap) mass analyzer, respectively. Melting points were determined in open glass capillary with a Stuart scientific SMP3 apparatus.

Synthesis of isocyanide 8. Amino alcohol (20.00 mmol, 1 equiv) 3 and ethyl formate (13 mL) were heated under reflux for six hours. The volatile was removed under vacuo. The corresponding formamide (7.50 mmol, 1 equiv) was solubilized in dry $\mathrm{CH}_{2} \mathrm{Cl}_{2}(14 \mathrm{~mL})$ under nitrogen and, after adding TEA (45.00 mmol, 6 equiv), the reaction mixture was cooled at $0{ }^{\circ} \mathrm{C}$. Then, $\mathrm{TsCl}(22.50 \mathrm{mmol}$, 3 equiv) was added and the reaction was stirred at room temperature for 5 hours. The mixture was quenched with a saturated aqueous $\mathrm{Na}_{2} \mathrm{CO}_{3}$ solution and stirred under cooling at $0{ }^{\circ} \mathrm{C}$ for 30 minutes. Water was added and the aqueous phase was extracted with $\mathrm{CH}_{2} \mathrm{Cl}_{2}(\mathrm{x} 3)$. The organic layer was dried over sodium sulfate and evaporated. The crude product was purified by silica gel column 
chromatography using PE/EtOAc 9:1, affording compound $\mathbf{8}(1.14 \mathrm{~g}, 57 \%)$ as a yellow oil. See the Supplementary Information for a complete characterization.

Synthesis of thalidomide-bearing isocyanide 14. To a solution of thalidomide derivative $11(6.00$ mmol, 1 equiv) in dry DMF (25.50 mL), $\mathrm{NaHCO}_{3}(12.00 \mathrm{mmol}, 2$ equiv) was added under nitrogen and the reaction mixture was heated at $80{ }^{\circ} \mathrm{C}$. After 15 minutes a solution of isocyanide 8 (10.20 mmol, 1.7 equiv) was added dropwise and the resulting mixture was stirred at $80{ }^{\circ} \mathrm{C}$ overnight. Then, it was diluted with dichloromethane and washed with water (x3). The organic layer was dried over sodium sulfate and the volatile was removed under vacuo. The crude product was purified by silica gel column chromatography using PE/EtOAc 5:5 as eluent, affording compound $\mathbf{1 4}(1.79 \mathrm{~g}, 81 \%)$ as a white solid. See the Supplementary Information for a complete characterization.

Synthesis of PROTAC 34 by split Ugi reaction. To a solution of piperazine 31 ( $0.12 \mathrm{mmol}, 1$ equiv) in $\mathrm{CH}_{3} \mathrm{OH}(0.5 \mathrm{~mL})$ paraformaldehyde $\mathbf{1 8}(0.12 \mathrm{mmol}, 1$ equiv), isocyanide 14 (0.12 mmol, 1 equiv) and carboxylic acid 17 (0.12 mmol, 1 equiv) were added sequentially. The reaction mixture was heated at reflux for 2 hours, then the volatile was removed under vacuo and the crude product was purified by silica gel column chromatography using $\mathrm{CH}_{3} \mathrm{CN} / \mathrm{MeOH}$ 9:1 as eluent, affording compound $34(78.1 \mathrm{mg}, 75 \%)$ as a white solid. See the Supplementary Information for a complete characterization.

\section{Acknowledgements}

M. S. is supported by Fondazione AIRC (Associazione Italiana per la Ricerca sul Cancro) fellowship for Abroad (Rif. 25278). T.P. holds a grant from Ministero dell'Istruzione, dell'Università e della Ricerca (MIUR) (PRIN $2017 \mathrm{~N}^{\circ}$ 2017WJZ9W9). This project has received funding from the European Union's Horizon 2020 Research and Innovation Programme under the grant agreement No. 956477. The authors acknowledge Dr. Gianluca Papeo for manuscript proofreading and Prof. Gian Cesare Tron for insightful suggestions. 


\section{Author Contributions}

M.S. and T.P. conceived and designed the experiments. I.P.B., A.R. and M.S. performed the experiments. I.P.B., A.R. and T.P. analysed the experimental data. M.S. and T.P. wrote the manuscript with input from all authors. All of the authors have approved the final version of the manuscript.

\section{References}

1. Lai, A. C. \& Crews, C. M. Induced protein degradation: an emerging drug discovery paradigm. Nat. Rev. Drug Discov. 16, 101-114 (2017).

2. Sakamoto, K. M. et al. Protacs: Chimeric molecules that target proteins to the Skp1-Cullin-F box complex for ubiquitination and degradation. Proc. Natl. Acad. Sci. 98, 8554-8559 (2001).

3. Bondeson, D. P. et al. Catalytic in vivo protein knockdown by small-molecule PROTACs. Nat. Chem. Biol. 11, 611-617 (2015).

4. Xie, T. et al. Pharmacological targeting of the pseudokinase Her3. Nat. Chem. Biol. 10, 1006$1012(2014)$

5. Mullard, A. Targeted protein degraders crowd into the clinic. Nat. Rev. Drug Discov. 20, 247$250(2021)$.

6. Dale, B. et al. Advancing targeted protein degradation for cancer therapy. Nat. Rev. Cancer (2021) doi:10.1038/s41568-021-00365-X.

7. Petrylak, D. P. et al. First-in-human phase I study of ARV-110, an androgen receptor (AR) PROTAC degrader in patients (pts) with metastatic castrate-resistant prostate cancer (mCRPC) following enzalutamide (ENZ) and/or abiraterone (ABI). J. Clin. Oncol. 38, 3500-3500 (2020).

8. Winter, G. E. et al. Phthalimide conjugation as a strategy for in vivo target protein degradation. Science 348, 1376-1381 (2015).

9. Gadd, M. S. et al. Structural basis of PROTAC cooperative recognition for selective protein degradation. Nat. Chem. Biol. 13, 514-521 (2017). 
10. Nowak, R. P. et al. Plasticity in binding confers selectivity in ligand-induced protein degradation. Nat. Chem. Biol. 14, 706-714 (2018).

11. Hughes, S. J. \& Ciulli, A. Molecular recognition of ternary complexes: a new dimension in the structure-guided design of chemical degraders. Essays Biochem. 61, 505-516 (2017).

12. Zaidman, D., Prilusky, J. \& London, N. PRosettaC: Rosetta Based Modeling of PROTAC Mediated Ternary Complexes. J. Chem. Inf. Model. 60, 4894-4903 (2020).

13. Bai, N. et al. Rationalizing PROTAC-Mediated Ternary Complex Formation Using Rosetta. $J$. Chem. Inf. Model. 61, 1368-1382 (2021).

14. Goracci, L. et al. Understanding the metabolism of proteolysis targeting chimeras (PROTACs): The next step toward pharmaceutical applications. J. Med. Chem. 63, 11615-11638 (2020).

15. Pike, A., Williamson, B., Harlfinger, S., Martin, S. \& McGinnity, D. F. Optimising proteolysistargeting chimeras (PROTACs) for oral drug delivery: a drug metabolism and pharmacokinetics perspective. Drug Discov. Today 25, 1793-1800 (2020).

16. Edmondson, S. D., Yang, B. \& Fallan, C. Proteolysis targeting chimeras (PROTACs) in 'beyond rule-of-five' chemical space: Recent progress and future challenges. Bioorg. Med. Chem. Lett. 29, 1555-1564 (2019).

17. Troup, R. I., Fallan, C. \& Baud, M. G. J. Current strategies for the design of PROTAC linkers: a critical review. Explor. Target. Anti-Tumor Ther. 1, 273-312 (2020).

18. Bemis, T. A., La Clair, J. J. \& Burkart, M. D. Unraveling the role of linker design in proteolysis targeting chimeras. J. Med. Chem. 64, 8042-8052 (2021).

19. Bricelj, A., Steinebach, C., Kuchta, R., Gütschow, M. \& Sosič, I. E3 ligase ligands in successful PROTACs: An overview of syntheses and linker attachment points. Front. Chem. 9, 707317 (2021).

20. Cecchini, C., Pannilunghi, S., Tardy, S. \& Scapozza, L. From conception to development: investigating PROTACs features for improved cell permeability and successful protein degradation. Front. Chem. 9, 672267 (2021). 
21. Steinebach, C. et al. PROTAC-mediated crosstalk between E3 ligases. Chem. Commun. Camb. Engl. 55, 1821-1824 (2019).

22. Krajcovicova, S., Jorda, R., Hendrychova, D., Krystof, V. \& Soural, M. Solid-phase synthesis for thalidomide-based proteolysis-targeting chimeras (PROTAC). Chem. Commun. 55, 929-932 (2019).

23. Wurz, R. P. et al. A 'click chemistry platform' for the rapid synthesis of bispecific molecules for inducing protein degradation. J. Med. Chem. 61, 453-461 (2018).

24. Bemis, T. A., La Clair, J. J. \& Burkart, M. D. Traceless Staudinger ligation enabled parallel synthesis of proteolysis targeting chimera linker variants. Chem. Commun. Camb. Engl. 57, 1026-1029 (2021).

25. Brownsey, D. K., Rowley, B. C., Gorobets, E., Gelfand, B. S. \& Derksen, D. J. Rapid synthesis of pomalidomide-conjugates for the development of protein degrader libraries. Chem. Sci. 12, 4519-4525 (2021).

26. Serafini, M., Pirali, T. \& Tron, G. C. Chapter Three - Click 1,2,3-triazoles in drug discovery and development: From the flask to the clinic? in Advances in Heterocyclic Chemistry (eds.

Meanwell, N. A. \& Lolli, M. L.) vol. 134 101-148 (Academic Press, 2021).

27. Ugi, I., Meyr, R., Fetzer, U., Steinbrückner, C. Versammlungsberichte. Angew. Chem. 71, 373388 (1959).

28. Ahmed Fouad, M., Abdel-Hamid, H. \& Salah Ayoup, M. Two decades of recent advances of Ugi reactions: synthetic and pharmaceutical applications. RSC Adv. 10, 42644-42681 (2020) and references herein.

29. Waibel, K. A., Nickisch, R., Möhl, N., Seim R., \& Meier, M. A. R. A more sustainable and highly practicable synthesis of aliphatic isocyanides. Green Chem. 22, 933-941 (2020)

30. The synthesized isocyanides were stored at room temperature in closed vials and after one month the NMR spectra did not . 
31. Bhela, I. P., Serafini, M., Del Grosso, E., Tron, G. C. \& Pirali, T. Tritylamine as an ammonia surrogate in the Ugi reaction provides access to unprecedented 5-sulfamido oxazoles using Burgess-type reagents. Org. Lett. 23, 3610-3614 (2021).

32. Giovenzana, G. B., Tron, G. C., Di Paola, S., Menegotto, I. G. \& Pirali, T. A mimicry of primary amines by bis-secondary diamines as components in the Ugi four-component reaction. Angew. Chem. Int. Ed Engl. 45, 1099-1102 (2006).

33. Alabi, S. et al. Mutant-selective degradation by BRAF-targeting PROTACs. Nat. Commun. 12, $920(2021)$.

34. Farnaby, W. et al. BAF complex vulnerabilities in cancer demonstrated via structure-based PROTAC design. Nat. Chem. Biol. 15, 672-680 (2019).

35. Han, X. et al. Discovery of ARD-69 as a highly potent proteolysis targeting chimera (PROTAC) degrader of androgen receptor (AR) for the treatment of prostate cancer. J. Med. Chem. 62, 941964 (2019).

36. Arvinas unveils PROTAC structures. Chemical \& Engineering News https://cen.acs.org/pharmaceuticals/drug-discovery/Arvinas-unveils-PROTAC-structures/99/i14.

37. Klein, V., Bond, A., Craigon, C., Lokey, R. S. \& Ciulli, A. Amide-to-ester substitution as a strategy for optimizing PROTAC permeability and cellular activity. Preprint at: doi:10.33774/chemrxiv-2021-47tqn (2021).

38. Passerini, M.; Simone, L. Sopra gli isonitrili (I). Composto del p-isonitril-azobenzolo con acetone ed acido acetico. Gazz. Chim. Ital. 51, 126-129 (2021).

39. Neklesa, T. K. et al. Small-molecule hydrophobic tagging-induced degradation of HaloTag fusion proteins. Nat. Chem. Biol. 7, 538-543 (2011).

40. Long, M. J. C., Gollapalli, D. R. \& Hedstrom, L. Inhibitor mediated protein degradation. Chem. Biol. 19, 629-637 (2012).

41. Neklesa, T. K. \& Crews, C. M. Greasy tags for protein removal. Nature 487, 308-309 (2012). 
42. Stucchi, M. \& Lesma, G. Split-Ugi reaction with chiral compounds: Synthesis of piperazineand bispidine-based peptidomimetics. Helv. Chim. Acta 99, 315-320 (2016).

43. Morana, F., Basso, A., Riva, R., Rocca, V. \& Banfi, L. The homo-PADAM protocol: stereoselective and operationally simple synthesis of $\alpha$-oxo- or $\alpha$-hydroxy- $\gamma$-acylaminoamides and chromanes. Chem. Weinh. Bergstr. Ger. 19, 4563-4569 (2013).

44. Zheng, M. et al. Rational Design and synthesis of novel dual PROTACs for simultaneous degradation of EGFR and PARP. J. Med. Chem. 64, 7839-7852 (2021).

45. Imaide, S. et al. Trivalent PROTACs enhance protein degradation through cooperativity and avidity. Preprint at: doi:10.26434/chemrxiv.13218695.v1 (2020).

46. The average cost of the top-selling building blocks is 300 euros per $50 \mathrm{mg}$; https://www.sigmaaldrich.com/IT/it/technical.

47. Bradner, J., Buckley, D. \& Winter, G. Methods to Induce Targeted Protein Degradation Through Bifunctional Molecules. WO Patent 2017007612 A1 (2017).

48. Nowak, R. P. et al. Heterobifunctional Compounds with Improved Specificity for the Bromodomain of Brd4. WO Patent 2019079701 A1 (2019). 